\title{
Living reactions of tissue-engineered bone derived from apatite-fiber scaffold in rat subcutaneous tissues
}

\author{
Kitaru SUZUKI ${ }^{1}$, Michiyo HONDA ${ }^{1,2}$, Tomokazu MATSUURA ${ }^{3}$ and Mamoru AIZAWA ${ }^{1,2, \dagger}$ \\ ${ }^{1}$ Department of Applied Chemistry, School of Science and Technology, Meiji University, \\ 1-1-1 Higashimita, Tama-ku, Kawasaki 214-8571, Japan \\ ${ }^{2}$ Meiji University International Institute for Materials with Life Functions, Meiji University, \\ 1-1-1 Higashimita, Tama-ku, Kawasaki 214-8571, Japan \\ ${ }^{3}$ Department of Laboratory Medicine, The Jikei University School of Medicine, \\ 3-25-8 Nishi-shinbashi, Minato-ku, Tokyo 105-8461, Japan
}

\begin{abstract}
We synthesized apatite fibers (AFs) and fabricated a porous hydroxyapatite scaffold (apatite-fiber scaffold; AFS). A tissue-engineered bone involving a three-dimensional structure was constructed by placing AFS in a radial-flow bioreactor (RFB) to culture rat bone marrow mesenchymal stem cells (RBMCs). In this study, we examined whether the tissue-engineered bone derived from the AFS has osteogenic differentiation potential leading to bone-forming ability in vivo in the subcutaneous tissue where bone formation does not occur naturally due to the absence of osteoblasts. The reconstructed tissue-engineered bone was implanted subcutaneously in rat tissue for 4 weeks. The AFS alone was implanted as a control. After implantation, some histological evaluations, i.e., hematoxylin and eosin (HE), alkaline phosphatase (ALP) staining, and immunofluorescence staining for osteocalcin (OC), were performed for the harvested samples. In addition, quantitative evaluation was also performed to determine the ALP activity normalized for the DNA content of the harvested samples. The HE staining revealed that both AFSs (control) and implanted tissue-engineered bone (RFB-bone) were biocompatible, and did not induce inflammation or immunological rejection in vivo. The ALP activity in the RFB-bone was significantly higher than that in the control. Immunofluorescent staining showed the expression of $\mathrm{OC}$ in RFB-bone. Therefore, we conclude that the tissue-engineered bone derived from AFS may have the boneforming ability in vivo in the absence of osteoblasts. These results may provide valuable insights into the design of tissue-engineered bone for clinical applications.

(02022 The Ceramic Society of Japan. All rights reserved.
\end{abstract}

Key-words: Hydroxyapatite, Apatite-fiber scaffold, Rat bone marrow mesenchymal cell, Tissue-engineered bone, Radial-flow bioreactor

[Received July 8, 2021; Accepted August 27, 2021]

\section{Introduction}

Bone grafts are required in the treatment of bone defects caused by trauma and various diseases. Autologous bone graft is a gold standard in the treatment for repairing bone tissue damage. ${ }^{1)}$ This approach involves osteoinduction, which is important for bone defect treatment. ${ }^{2)}$ Although autografts are the preferred option in clinical applications, two major autografting-associated problems remain to be addressed: limited amount of the grafted bone and secondary invasion of the healthy bone tissue. ${ }^{1)}$ Although allografts can solve both of these problems, they may lead to graft rejection or infection. ${ }^{3)}$ Traditional treatments,

Corresponding author: M. Aizawa; E-mail: mamorua@ meiji.ac.jp

$\$$ Preface for this article: Dol http://doi.org/10.2109/jcersj2. 130.P1-1 such as autologous bone grafts also result in various complications. ${ }^{4)}$ To avoid such problems, the development of artificial bones is being explored with promising results in recent years.

Tissue engineering comprises a combination of three components for the reconstruction of the target tissue: (i) cells, (ii) scaffold, and (iii) growth factors. Hydroxyapatite $\left[\mathrm{Ca}_{10}\left(\mathrm{PO}_{4}\right)_{6}(\mathrm{OH})_{2} ; \mathrm{HAp}\right]$ is a key inorganic component of biological hard tissues, that has biocompatibility and osteoconductivity. ${ }^{5)}$ It has been used clinically as bioceramics in bone grafting for scaffold material. ${ }^{6)-8)}$

Tissue engineering has also been successfully applied to bone reconstruction in recent years, and it can even repair tissues that are difficult to treat with materials alone. The reconstructed tissue might function effectively if it mimics the original three-dimensional (3D) structure of the replaced bone tissue. There are several reports about scaffolds made using a 3D printer in clinical applications. ${ }^{9)}$ 
For the bone tissue reconstruction, the size and connectivity of pores in the scaffold are important factors in scaffold material design, as their performance affects boneforming ability. ${ }^{10)}$ Therefore, porous scaffolds are often used for bone reconstruction. Using tissue engineering technique, we successfully constructed a tissue-engineered bone using (i) HAp porous scaffold, (ii) radial-flow bioreactor (RFB), and (iii) rat bone marrow mesenchymal stem cells (RBMCs). ${ }^{11)}$

Yoshikawa et al. ${ }^{12)}$ reported the reconstruction of bone tissue combining mesenchymal stem cells collected from bone marrow and porous HAp ceramics. The authors discussed there are two problems in reconstructing bone tissue: (i) a low number of mesenchymal stem cells in bone marrow, (ii) long time required by the cells to express osteoblast phenotype. Therefore, the authors devised a method to accelerate the bone formation and advocated it as the ideal one. In this method, the bone marrow cells were allowed to proliferate in vitro environment and then induced to undergo osteodifferentiation. These preosteodifferentiated cells were then seeded on the scaffold, which led to rapid in vivo bone formation. In order to develop optimum scaffolds for bone reconstruction, we synthesized single-crystal apatite fibers (AFs) by a homogeneous precipitation method using urea. ${ }^{13)}$ Using these AFs, we successfully developed porous apatite-fiber scaffolds (AFSs), which have 3D inter-connected pores of diameter $110-250 \mu \mathrm{m}$ and high porosities of $98-99 \%$. We also constructed a 3D tissue-engineered bone using AFS and an RFB with RBMCs but its bone-forming ability in vivo needed to be confirmed. Thus, we first examined the properties of RFB culture concerning cell invasiveness and osteodifferentiation under the two culture environments. To assess its clinical application, tissue-engineered bone made using RFB was implanted into rat subcutaneous tissue in absence of osteoblasts. The subcutaneous tissue is one of the places where bone formation does not take place naturally, as there are few cells involved in bone formation. Therefore, we focused on examining the osteo- genic differentiation potential of tissue-engineered bone alone by means of subcutaneous implantation model. After 4 weeks, the tissue-engineered bone was evaluated for osteogenic differentiation potential in vivo.

\section{Experimental procedure}

\subsection{Fabrication of AFSs and their characterization}

AFs were synthesized as per earlier report. ${ }^{13), 14)}$ The following aqueous solutions were prepared: 0.167 $\mathrm{mol} \cdot \mathrm{dm}^{-3} \mathrm{Ca}\left(\mathrm{NO}_{3}\right)_{2}, 0.100 \mathrm{~mol} \cdot \mathrm{dm}^{-3}\left(\mathrm{NH}_{4}\right)_{2} \mathrm{HPO}_{4}, 0.500$ $\mathrm{mol} \cdot \mathrm{dm}^{-3}\left(\mathrm{NH}_{2}\right)_{2} \mathrm{CO}$, and $0.10 \mathrm{~mol} \cdot \mathrm{dm}^{-3} \mathrm{HNO}_{3}$. The $\mathrm{Ca} / \mathrm{P}$ ratio was adjusted to 1.67 . Figure 1 shows illustration of the preparation process of AFS2000 together with the overview. The AFs were mixed with spherical carbon beads of $\sim 150 \mu \mathrm{m}$ diameter in a mixed solvent (ethanol/ water $=1: 1[\mathrm{v} / \mathrm{v}]$ ) with an $\mathrm{AF} /$ carbon bead $[\mathrm{w} / \mathrm{w}]$ ratio of $1 / 20 .{ }^{15)}$ The green compacts for scaffolds were fabricated by pouring the carbon beads containing AF slurry into a vinyl chloride tube $(\sim \phi 20 \mathrm{~mm})$ mold. A glass tube (outer diameter $\sim 7 \mathrm{~mm}$ ) was placed at the center of the vinyl chloride tube mold, and the slurry containing AFs and carbon beads $\left(18 \mathrm{~cm}^{3}\right)$ was poured with a micropipette and then vacuumed by a pump. ${ }^{16)}$ Further, compacts were fired at $1300{ }^{\circ} \mathrm{C}$ for $5 \mathrm{~h}$ in steam atmosphere to produce AFSs, referred to as "AFS2000". In this study, we used AFS2000 with a diameter of $\sim \phi 18 \mathrm{~mm}$ and a height of $\sim 15 \mathrm{~mm}$. The identity of crystalline phase of AFs and AFS2000 were determined by X-ray diffractometer (XRD; Miniflex, Rigaku, Japan) with a $\mathrm{Cu} \mathrm{K}_{\alpha}$ radiation source at $30 \mathrm{kV}$ and $15 \mathrm{~mA}$, and the data was collected in the $2 \theta$ range of 4 $50^{\circ}$ at scan rate of $4^{\circ}$ per minute, and sampling width of $0.04^{\circ}$. The functional groups of AFs and AFS2000 were detected using Fourier transforms infrared spectrometry (FT-IR; IR Prestige-21, Shimadzu, Japan) in the range 400$4000 \mathrm{~cm}^{-1}$ with a spectral resolution of $4 \mathrm{~cm}^{-1}$. The pellets for FT-IR measurements were prepared by compressing the sample mixed with $\mathrm{KBr}$ powders. The particle morphologies of AFs were observed by scanning electron micros-
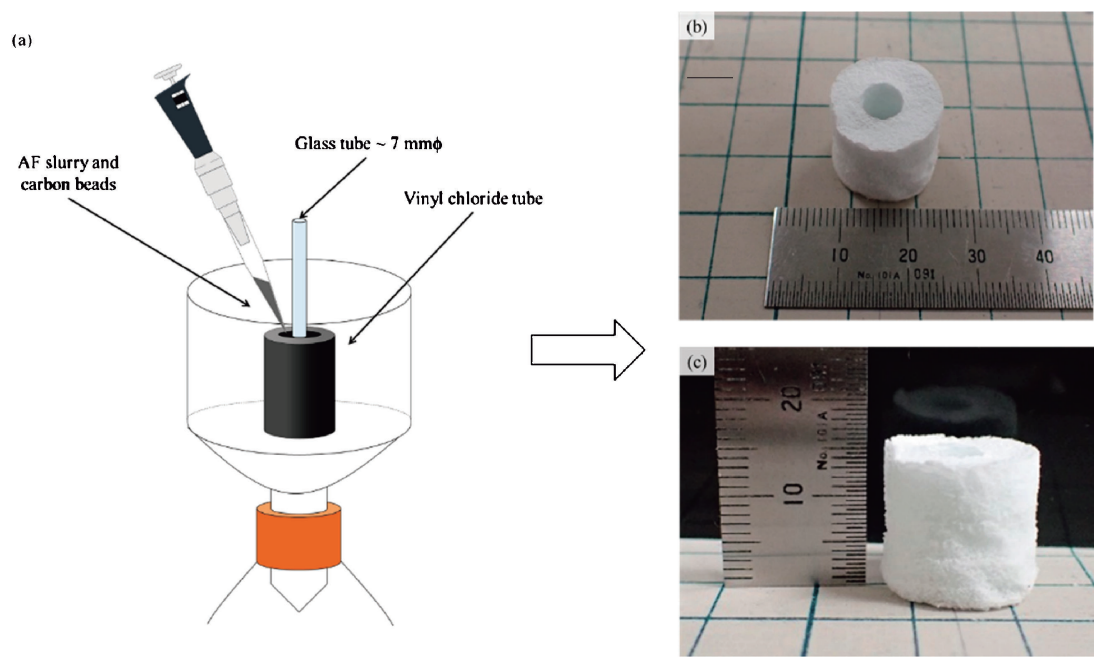

Fig. 1. Illustration of fabrication method and photographs of AFS2000: (a) fabrication scheme, (b) top, and (c) side views. 
copy (SEM; JSM6390LA, JEOL, Japan) at $10 \mathrm{kV}$, together with the microstructure of AFSs. SEM samples were prepared by fixing the AFs and AFSs on double-sided carbon tapes and depositing platinum particles in a vacuum.

\subsection{Primary culture of RBMCs}

RBMCs were obtained from the bone marrow of femora and tibiae from 4-week-old male Wistar rats, as previously reported. ${ }^{17)}$ Both ends of the femora and tibiae were cut away from the epiphysis, and marrows were flushed out with $\alpha$-minimal essential medium ( $\alpha$-MEM) using a syringe and 21-gage needle. Cells were harvested from bone shafts and cultured in a standard medium $[\alpha$-MEM with $10 \%$ fetal bovine serum and antibiotics (100 units $\cdot \mathrm{cm}^{-3}$ penicillin and $100 \mu \mathrm{g} \cdot \mathrm{cm}^{-3}$ streptomycin)] at $37^{\circ} \mathrm{C}$ in a humidified atmosphere with $5 \% \quad \mathrm{CO}_{2}$. The medium was changed after 2 days to remove unattached cells. The medium was changed every 2 days. After 8 days, RBMCs were harvested using $0.25 \%$ trypsin-EDTA solution (Gibco, Thermo Fisher Scientific, US) and a cell scraper (IWAKI, Japan). After 16 days, cells were removed from culture dishes using $0.25 \%$ trypsin-EDTA solution. The count of cells collected was $1.0 \times 10^{7}$. All the animal treatments were performed according to the Guidelines for Animal Care and Use Committee of Meiji University (No. MUIACUC2020-11).

\subsection{Reconstruction of tissue-engineered bone using RFB and their biological evaluations}

The AFS2000 was soaked in the standard medium and placed into an RFB a day before cell seeding. The RFB system (ABLE, Japan) with a chamber volume of $5 \mathrm{~cm}^{3}$ was used for the 3D-cell culture of RBMCs. Figure 2 shows the overview of RFB and schematic of medium flow in the bioreactor. The RBMCs suspended in the medium were injected into the RFB reservoir. The flow rate of the medium was set at an optimized flow rate at $6.3 \mathrm{~cm}^{3} \cdot \mathrm{min}^{-1}$ on the basis of our previous report, ${ }^{16)}$ and cells were seeded in/on AFSs. The cells were allowed to proliferate in the RFB in a standard medium for 1 week. This was followed by the cells in a differentiation medium (standard medium containing Osteoblast-inducer Reagent, TaKaRa, Japan) for 1 week. Thus, the tissue-engineered bone derived from the AFS2000 was constructed the 3Dcell culture technology.

To confirm the proliferation of RBMCs during 3D-cell culture, the levels of glucose and lactic acid in the medium were monitored using Glu Test Ace R (Arkray, Japan) and Lactate Pro (Arkray, Japan), respectively.

Histological evaluation for tissue-engineered bone before implantation was performed to determine bone differentiation properties. Tissue-engineered bone samples were fixed in $4 \%$ paraformaldehyde (PFA) in PBS for $60 \mathrm{~min}$ at $4{ }^{\circ} \mathrm{C}$. Next, the samples were immersed in $1.0 \%$ gelatin solution for $60 \mathrm{~min}$ at $25^{\circ} \mathrm{C}$ and kept in the same solution for $60 \mathrm{~min}$ under reduced pressure. Finally, the samples were embedded in an OCT compound (SAKURA TissueTek, Japan) and frozen at $-20^{\circ} \mathrm{C}$ overnight. Frozen specimens were then transferred to $-80^{\circ} \mathrm{C}$ until section preparation. Frozen sections with a thickness of $18 \mu \mathrm{m}$ were cut using a microtome (CM3050 S, Leica, Germany).

For the specimens, hematoxylin and eosin (HE) staining was performed to examine cellular localization. Specimens were soaked in HE solutions (SAKURA Tissue-Tek, Japan).

Alkaline phosphatase (ALP) staining was performed using Tartrate-Resistant Acid Phosphatase/ALP stain kit (Wako Pure Chemical Industry, Japan). The specimens were soaked in ALP premixed substrate solution, incubated at $37^{\circ} \mathrm{C}$ for $30 \mathrm{~min}$, then washed with sterile water.

For immunostaining, the specimens were permeabilized with $0.1 \%$ Triton X-100/PBS for $10 \mathrm{~min}$. They were then incubated in blocking buffer with $3 \%$ bovine serum albu$\min$ for $60 \mathrm{~min}$ at $25^{\circ} \mathrm{C}$ to block non-specific antibody binding. After removal of the blocking solution, the specimens were washed with PBS, and were then incubated with the primary anti-osteocalcin (OC) antibody (OC4-30, ab13418, Abcam, UK) overnight at $4{ }^{\circ} \mathrm{C}$. Further, the specimens were rinsed thoroughly and were incubated with a secondary antibody of Alexa Fluor 488 goat antimouse IgG2a antibody (A-21131, Thermo Fisher Scientific, US) for $1 \mathrm{~h}$ at $25^{\circ} \mathrm{C}$. The cell nuclei were stained with 4', 6-diamidino-2-phenylindole (DAPI, Dojindo, Japan).

Culturing of cells using RFB, used in this study, provides a "dynamic conditions." To gain insight into the effects of this dynamic environment on cell invasion and bone differentiation properties, we compared it to the

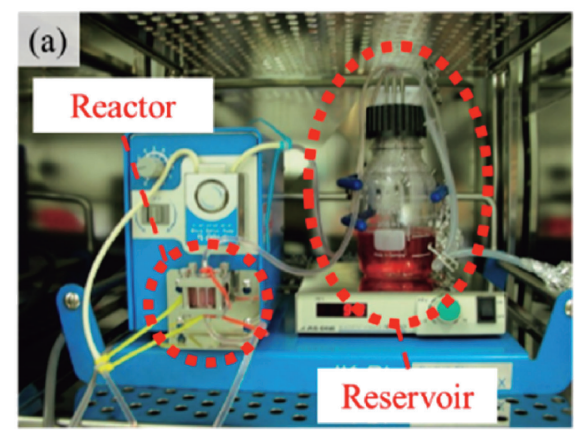

(b)

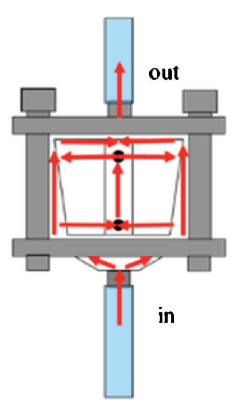

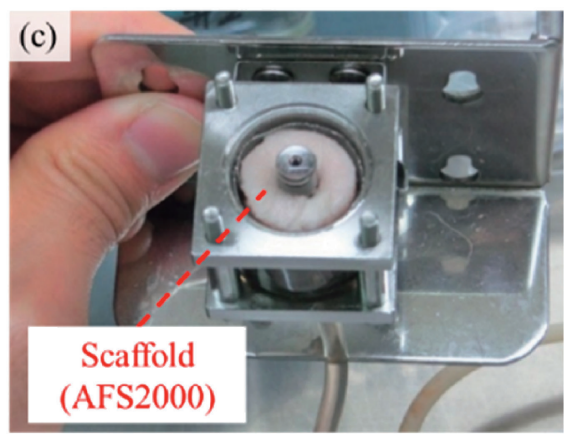

Fig. 2. Photographs of an RFB: (a) overview of an RFB, (b) scheme of medium flow in the bioreactor, and (c) AFS2000 placed in the bioreactor. 
reconstructed tissue-engineered bone in "static conditions". We cultured RBMCs with AFS2000 without using the RFB for static culture. The AFS2000 was soaked in the standard medium for 1 day before the seeding. RBMC cells $\left(1.0 \times 10^{7}\right)$ were seeded onto the soaked AFS2000 and cultured in a $50 \mathrm{~cm}^{3}$ centrifuge tube for 2 weeks. The fixation and evaluation were performed as described above.

For the histological evaluations, the specimens were imaged and analyzed using an optical microscope (BX51, Olympus, Japan). The stained sections were observed under the microscope to examine the cell proliferation, bone differentiation properties, and inflammation.

\subsection{Implantation of tissue-engineered bone for rat subcutaneous tissue}

All animal treatments and implantation procedures were approved by the Animal Research Committee of Meiji University. Seven-week-old male Wistar rats were anesthetized by isoflurane inhalation and divided into 2 experimental groups to study the effect of implants: (i) AFS2000 alone (control) and (ii) tissue-engineered bone constructed using RFB (RFB-bone). Before implantation, AFS2000 and tissue-engineered bone were divided into pieces of the one-eighth size of the original samples. AFS2000 was soaked in the standard medium for 1 day before implantation. Control and RFB-bone were implanted subcutaneously in rat tissue. The rats were sacrificed 4 weeks after the implantation to perform biological evaluations.

\subsection{Histological evaluations for implanted specimens}

After 4 weeks of implantation, harvested samples were washed in PBS and fixed using PFA. The fixing method was the same as described in Section 2.3. The frozen samples were divided into sections of about $10 \mu \mathrm{m}$ thickness. The specimens were subjected to HE, ALP, and immunofluorescent staining for $\mathrm{OC}$ as described in Section 2.3.

\subsection{Quantitative assay for implanted specimens}

Harvested samples were washed with $3 \mathrm{~cm}^{3}$ 4-(2hydroxyethyl)-1-piperazineethanesulfonic acid (HEPES) solution, and then centrifuged three times at $1000 \mathrm{rpm}$ for $5 \mathrm{~min}$. The washed samples were stored at $-80^{\circ} \mathrm{C}$ with $1 \mathrm{~cm}^{3}$ HEPES buffer until further processing for quantitative DNA evaluation. After thawing, the samples were homogenized using an ultrasonic homogenizer. The homogenized solutions were aliquoted $0.5 \mathrm{~cm}^{3}$ each, and $0.75 \mathrm{~cm}^{3} 10 \mathrm{mmol} \cdot \mathrm{dm}^{-3}$ EDTA solution ( $\mathrm{pH} 12.3$; adjusted with $\mathrm{NaOH}$ ) was added to $0.5 \mathrm{~cm}^{3}$ of the sample solution and incubated at $45^{\circ} \mathrm{C}$ for $3 \mathrm{~min}$. Subsequently, the solutions were centrifuged at $10000 \mathrm{rpm}$ for $3 \mathrm{~min}$, and $0.04 \mathrm{~cm}^{3}$ of $1 \mathrm{~mol} \cdot \mathrm{dm}^{-3} \mathrm{KH}_{2} \mathrm{PO}_{4}$ solution was added to $1 \mathrm{~cm}^{3}$ of the supernatant. The DNA amount was measured by mixing $0.75 \mathrm{~cm}^{3}$ of the fluorescent reagent (Hoechst 33258 ) solution and $0.75 \mathrm{~cm}^{3}$ of the sample solution. To clarify the bone differentiation properties, we determined the bone formation ability by performing an ALP activity assay and normalized it for DNA content for the remaining $0.5 \mathrm{~cm}^{3}$ sample solution. ALP activity was assessed by measuring absorption of $405 \mathrm{~nm}$ using LabAssay ALP kit (Wako Pure chemical Industry, Japan) according to the manufacturer's instructions.

\subsection{Statistical analysis}

In this study, the statistical analysis was performed using the Mann-Whitney U-test. After that, refer to the previous report, ${ }^{18)}$ Z-test was performed to determine the significant difference.

\section{Results and discussion}

\subsection{Characterization of AF and AFS2000}

XRD pattern and FT-IR spectra of AFs are shown in Figs. 3(a) and 3(b). The ICDD reference patterns (\#90432) were used to identify the crystalline phases for HAp. The XRD pattern showed the AFs were single-phase of HAp [Fig. 3(a)]. Additionally, the resulting XRD pattern indicated a strong 300 reflection peak comparable to the XRD pattern of HAp in ICDD. The FT-IR pattern spectra of AFs displayed bands at 3570, 1500-1350, 1200-900, $880-870,600$, and $570 \mathrm{~cm}^{-1}$ [Fig. 3(b)]. The absorptions assigned to the $\mathrm{PO}_{4}{ }^{3-}$ groups at 1200-900, 600, and 570 $\mathrm{cm}^{-1}$ and those assigned to the $\mathrm{OH}^{-}$group at $3570 \mathrm{~cm}^{-1}$ were detected. Moreover, the absorptions assigned to the $\mathrm{CO}_{3}{ }^{2-}$ were detected at $1500-1350$ and $880-870 \mathrm{~cm}^{-1}$. The AFs can be referred to as Type $\mathrm{AB}$ of $\mathrm{CO}_{3} \mathrm{HAp}$, as per previous report. ${ }^{13)}$ Morphology of AFs was observed by SEM [Fig. 3(c)]. The AFs consisted of fiber-shaped particles with a preferred orientation (300) corresponding to the $a$-plane of HAp. The XRD patterns revealed that singlephase HAp was presented in AFS [Fig. 3(d)]. FT-IR spectra of AFS also comprised of the characteristic functional groups of HAp structure without the $\mathrm{CO}_{3}{ }^{2-}$ peak [Fig. 3(e)]. The SEM images indicated that the AFS had two types of pores: (i) macropores of $\sim 150 \mu \mathrm{m}$ [Fig. 3(f)] and (ii) micropores formed by intertwining individual AFs [Fig. $3(\mathrm{~g})]$. The macropores were formed by burning carbon beads out from the green compact.

\subsection{Growth of RBMCs in AFSs using RFB}

When 3D-cell culture was performed using the RFB with AFSs, the amount of glucose consumption and lactate production in the culture medium were measured [Figs. 4(a) and 4(b)]. The amount of glucose consumption and lactate production increased through the entire culture period indicating that the cells proliferated on the scaffold utilizing the glucose and producing lactate during the process. ${ }^{19)}$ These results suggest that RBMCs can be successfully cultured in AFSs using RFB.

\subsection{Histological evaluations of tissue- engineered bone before implantation}

The specimens were prepared from the tissueengineered bone before implantation and were histologically examined with HE and ALP staining (Fig. 5). The 
(a)

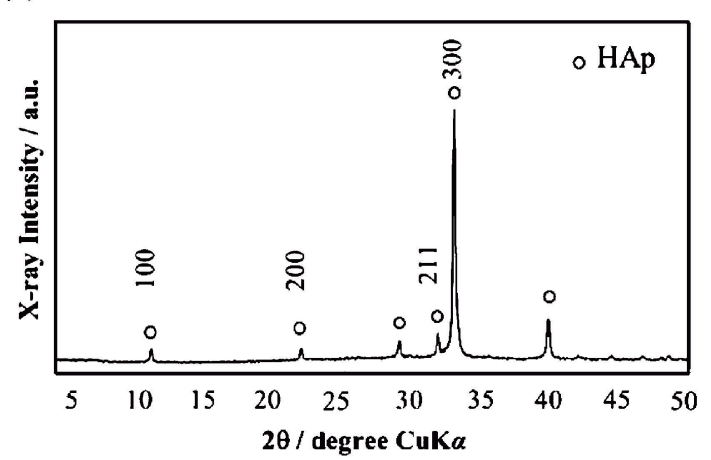

(b)

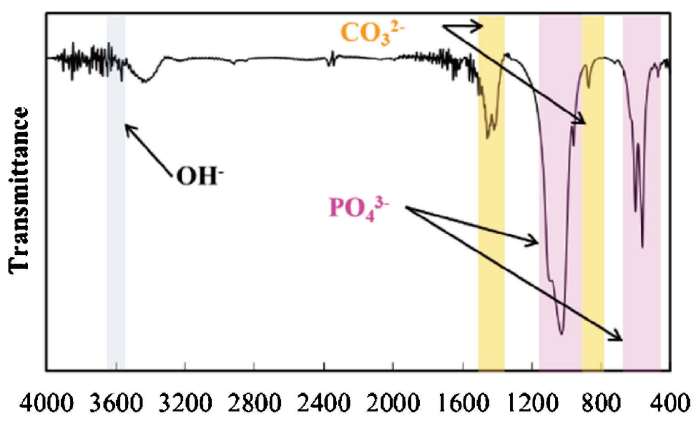

(c)

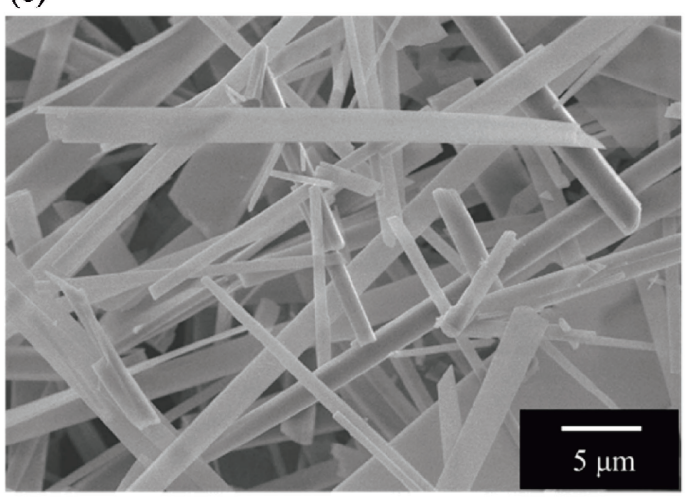

(d)

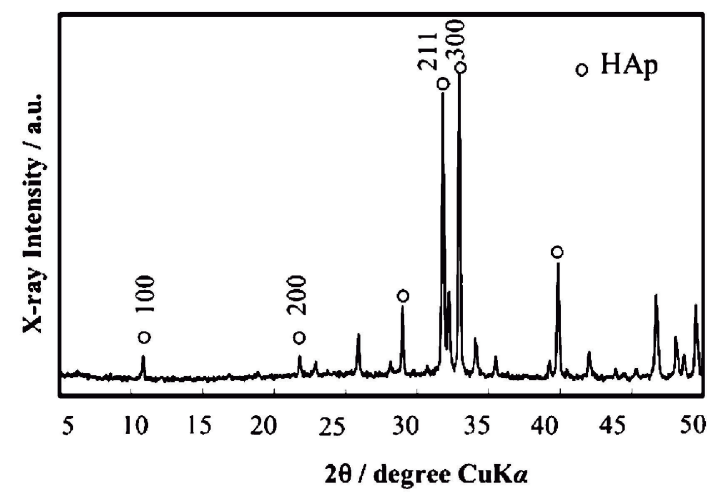

(f)

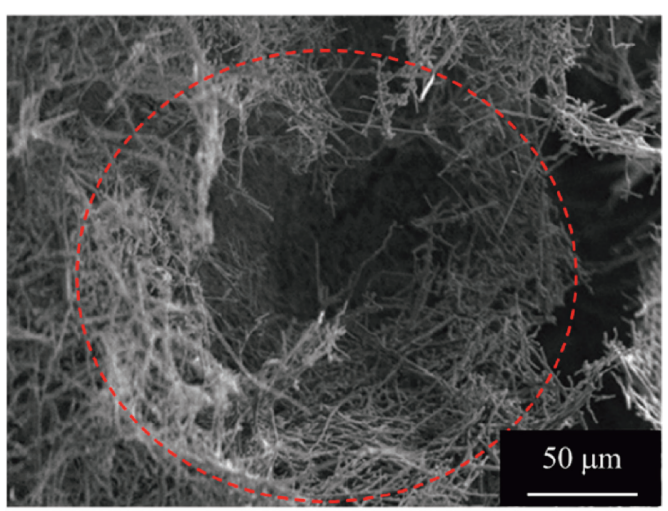

(e)

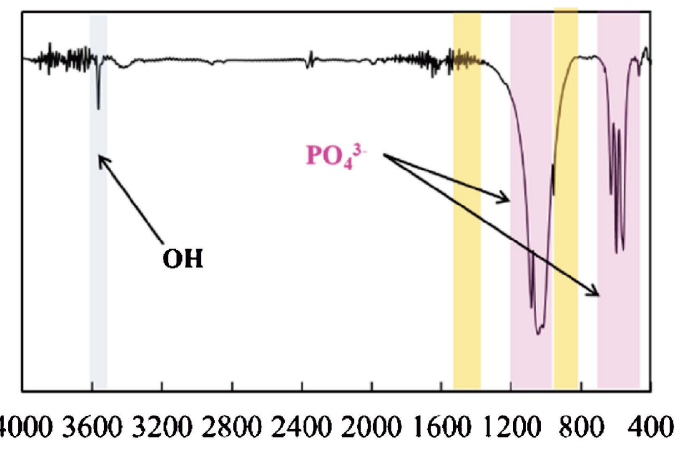

(g)

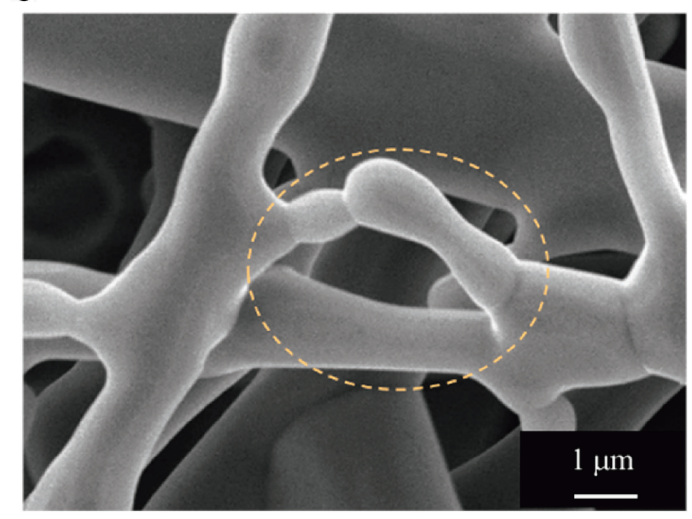

Fig. 3. Characterization of AFs and AFS2000: (a) XRD pattern, (b) FT-IR spectra, and (c) morphology of AFs; (d) XRD pattern, (e) FT-IR spectra, and (f) microstructure of AFS2000 at low and (g) high magnifications. Circle of red broken-line: Macropore. Circle of yellow broken-line: Micropore. 
(a)

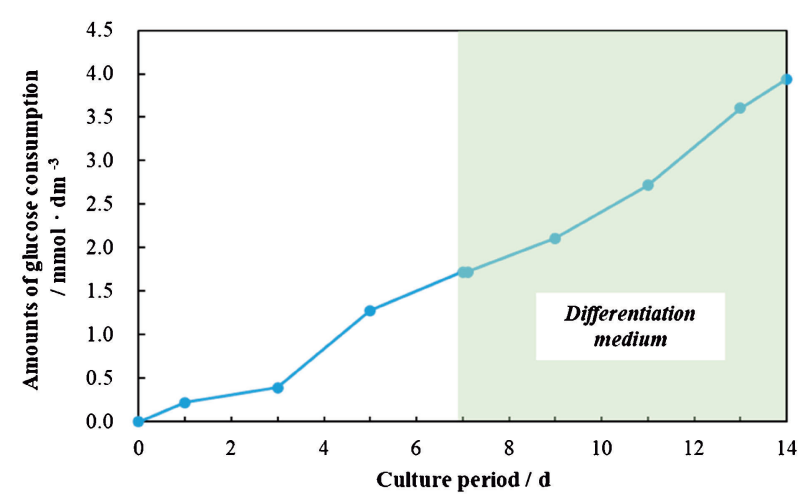

(b)

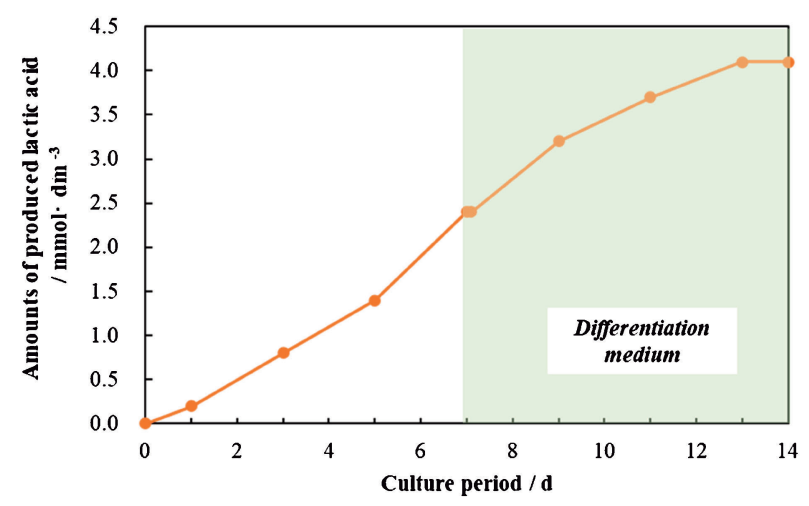

Fig. 4. Progress of 3D culture using RFB: (a) glucose consumption and (b) lactic acid production with culture period.

results of HE staining revealed that the cells in tissueengineered bone grew along with the AFS skeleton and were distributed throughout the fibrous scaffold [Fig. 5(a)]. However, the tissue-engineered bones cultured under "static conditions" had fewer cells at the bottom. In contrast, the cell distribution of the tissue-engineered bones cultured under "dynamic conditions" was relatively uniform [Fig. 5(b)].

The results of ALP staining showed ALP-positive cells (osteoblast-like cells) were present on the surface of the tissue-engineered bone [Fig. 5(a)]. Tissue-engineered bone culturing under "dynamic conditions" showed a high number of ALP-positive cells as compared to that under "static conditions" [Fig. 5(b)].

Immunofluorescence staining revealed a greater number of cells in the tissue-engineered bone constructed under "dynamic conditions" than that constructed under static ones (Fig. 6). The tissue-engineered bone under "dynamic conditions" showed high expression of OC while tissueengineered bone cultured under "static conditions" no OC expression. OC is synthesized and secreted by osteoblasts in the late stage of maturation. ${ }^{20)}$ The tissue-engineered bone constructed under "dynamic conditions" had a higher potential of osteodifferentiation than that in the case of "static conditions".

Overall, histological evaluations showed that tissueengineered bone cultured under "dynamic conditions" (a)

\section{Static culture (under static conditions)}

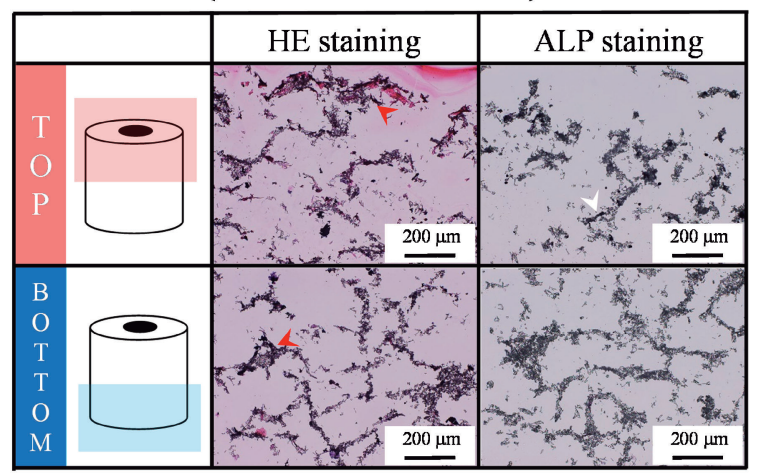

(b)

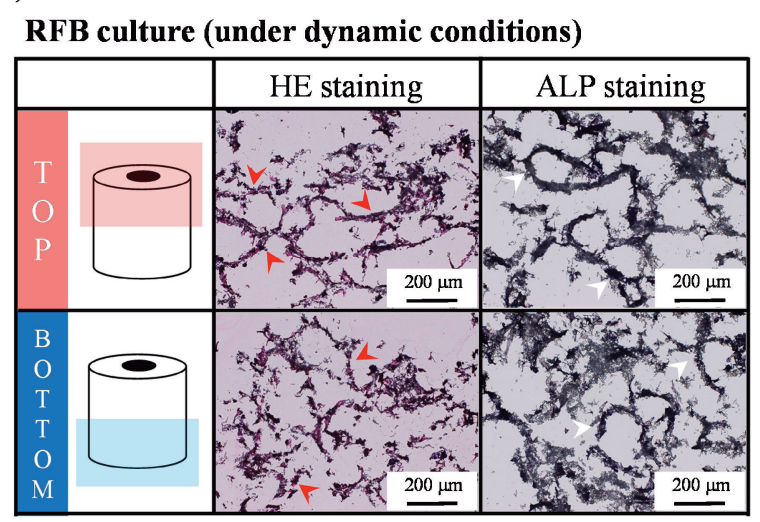

Fig. 5. Histological evaluation using HE and ALP staining for tissue-engineered bone: (a) under static conditions and (b) under dynamic conditions using RFB. Red arrowheads: Cells located along AFS skeletons. White arrowheads: ALP-positive cells.

using RFB possessed distributed RBMCs over the scaffold as compared to that cultured under "static conditions" (Figs. 5 and 6). Janssen et al. ${ }^{21}$ ) reported that the cell distribution was uniform on the scaffold when the tissueengineered bone was constructed using the perfusion bioreactor system. In addition, the authors assert that equal distribution of cells on the scaffold might decrease proliferation time. Results from the present study are in accordance with this report (Figs. 5 and 6). The dynamic environment may enable the cells to grow uniformly throughout the scaffold. In addition, the dynamic environment and uniform cell distribution in RFB offered favorable conditions for the cells to proliferate. Moreover, Schwartz et al. ${ }^{22}$ reported that the stimulus for increased expression of the osteogenic phenotype is either the improved nutrition with perfusion or the fluid flow and fluid deformation of the cells. Mechanical stimulation is also considered as one of the factors that promote bone differentiation. ${ }^{23)}$ Specimens cultured in a dynamic environment using RFB had higher ALP activity and OC expressing cells (Figs. 5 and 6). Therefore, the mechanical stimulation given by the circulation of the medium in RFB might have promoted bone differentiation. 


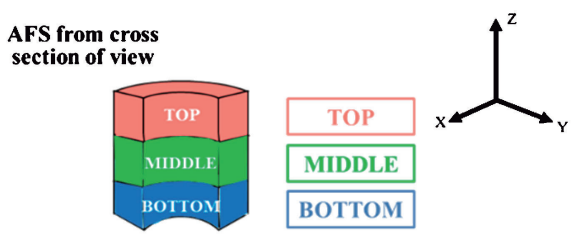

Observation of histological sections in the three area

(a) Static culture (under static conditions)
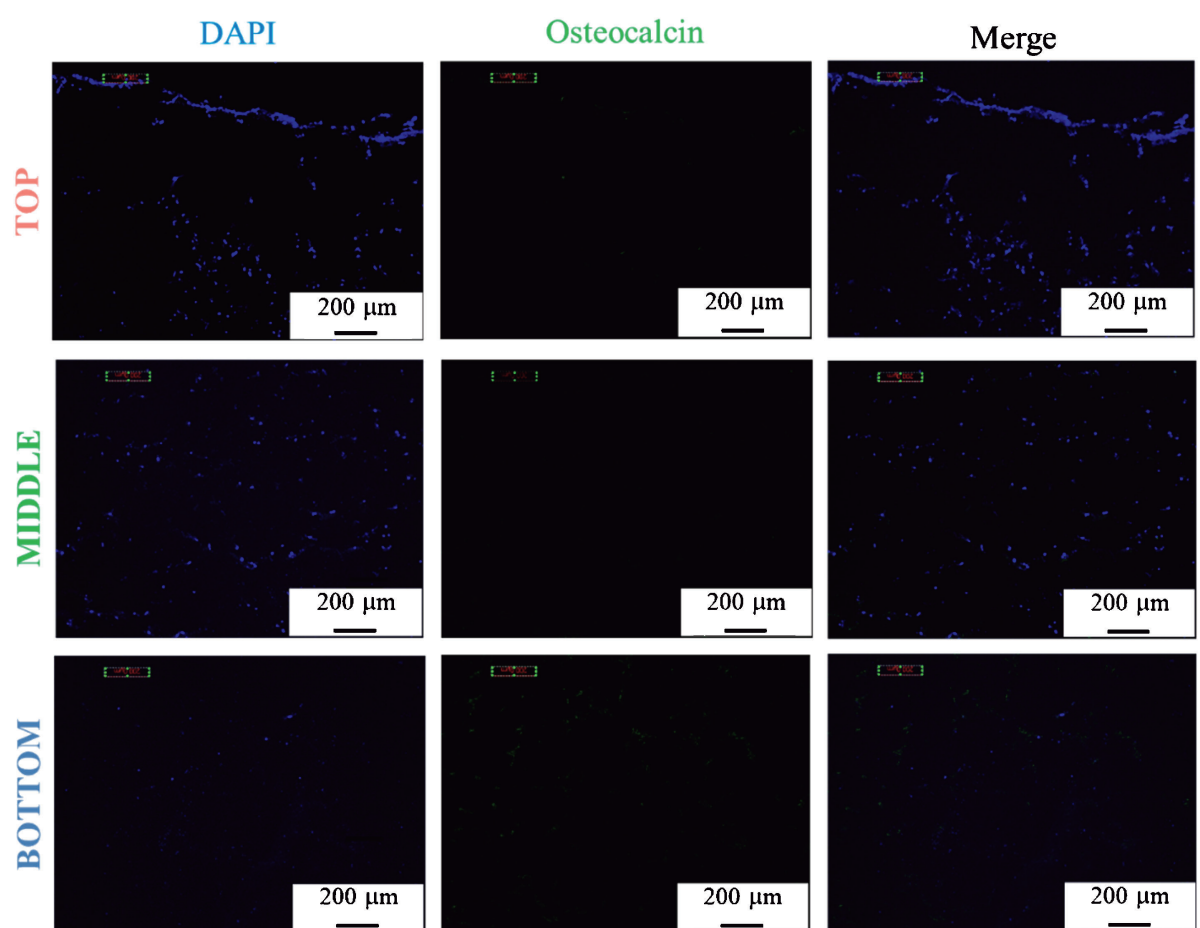

(b) RFB culture (under dynamic conditions)
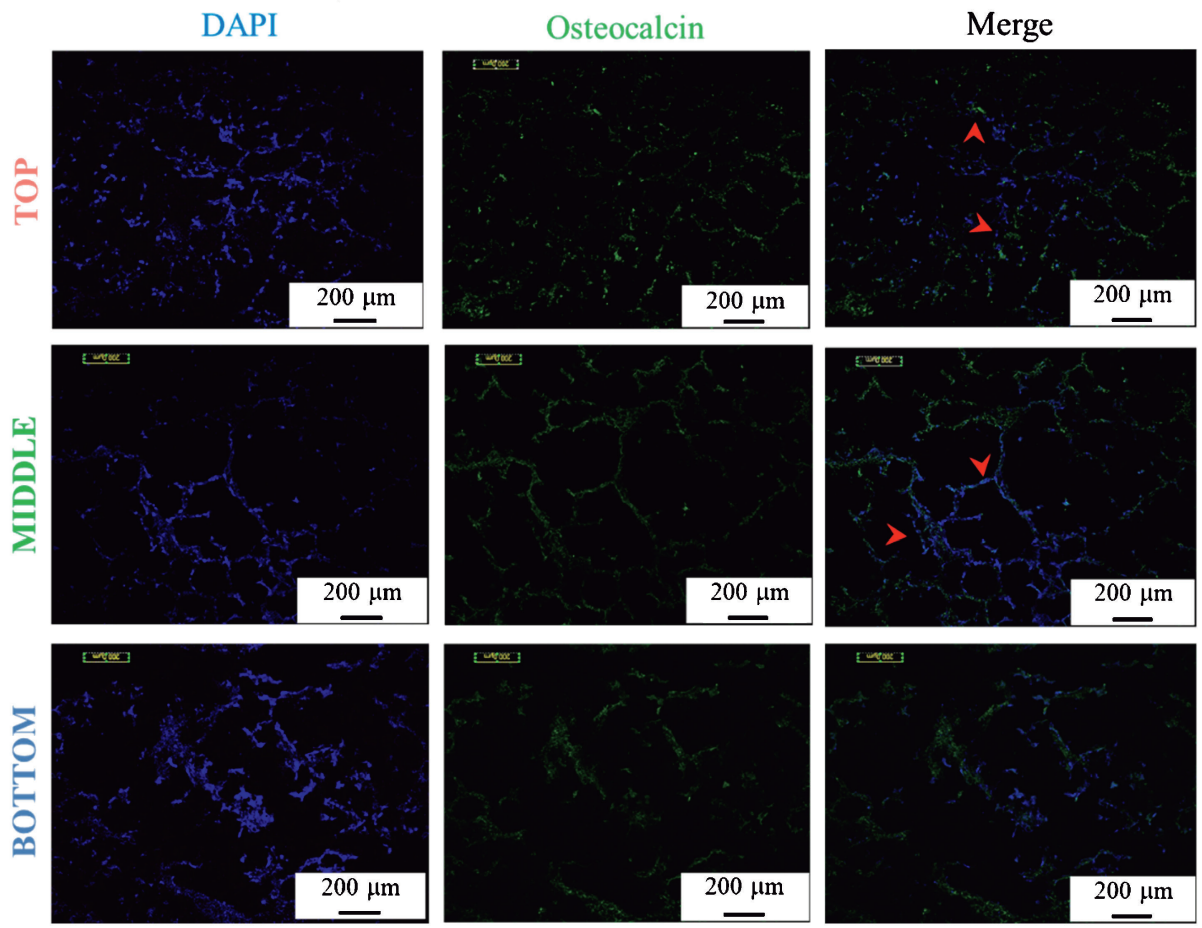

Fig. 6. Immunofluorescence staining of $\mathrm{OC}$ for tissue-engineered bone: (a) under static conditions and (b) under dynamic conditions using RFB. Red arrowheads: Cells expressing OC. 

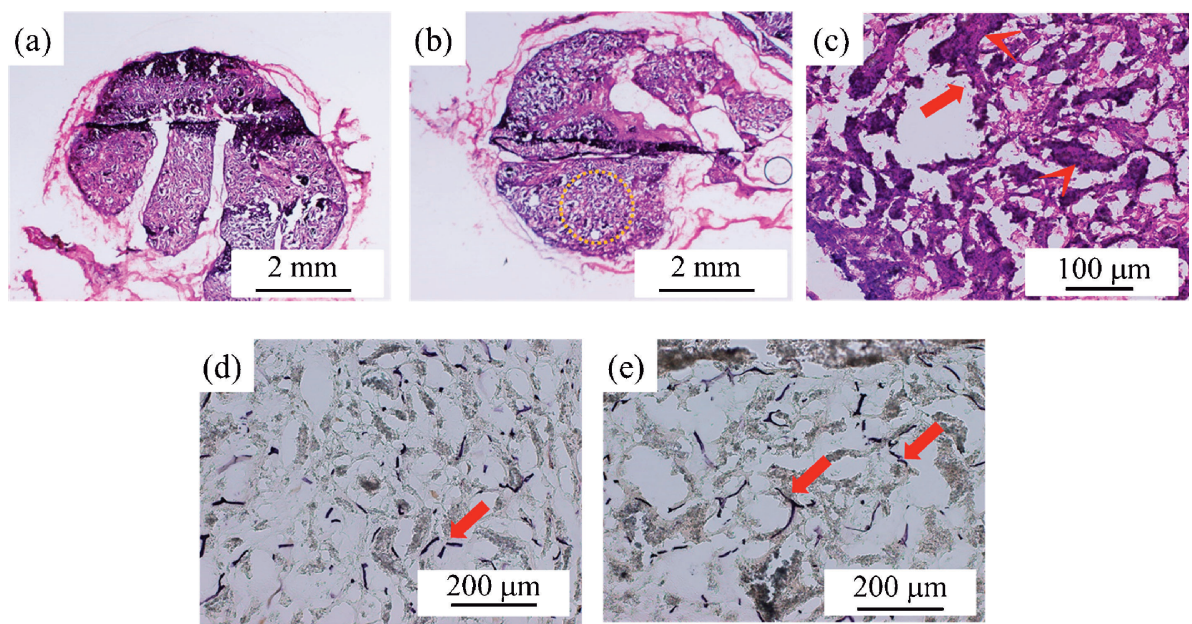

Fig. 7. Histological evaluation of specimens harvested from rat subcutaneous tissue: (a), (b), and (c)* HE staining (Red arrows and red arrow heads indicate skeletons of the AFS and the newly-formed bone tissue, respectively), (d) and (e) ALP staining (Red arrows indicate the ALP-positive cells), Sample groups: (a) and (d) control; (b), (c), and (e) tissue-engineered bone using RFB (RFB-bone). *: Enlarge the yellow circle of Fig. 7(b).

\subsection{Biological evaluations of implanted samples}

Subcutaneous implants were removed at after 4 weeks of implantation and histologically examined by $\mathrm{HE}$ and ALP staining. The results of HE staining showed that cells infiltrated into the AFSs [Figs. 7(a)-7(c)]. To maintain parts of the original shape, the implanted AFSs were not dissolved drastically. RFB-bone and control were covered with soft tissues. Moreover, no fibrous tissues were observed at the interface between the samples and subcutaneous tissue. These results indicate that AFS cultures do not induce significant inflammation or immunological reactivity, and have high biocompatibility in vivo. Furthermore, the red arrow heads in Fig. 7(c) might indicate the newly-bone tissue.

To validate the bone differentiation properties of tissueengineered bone under in vivo, the samples were examined with ALP staining [Figs. 7(d) and 7(e)]. As indicated by the red arrows, a higher number of ALP-positive cells were observed in the RFB-bone compared to that in control. In addition, ALP activity values normalized by the amount of DNA in each sample indicated significantly higher the ALP activity in the RFB-bone than the control samples (Fig. 8). Furthermore, immunofluorescent staining showed RFB-bone expressed OC as observed in vitro (Fig. 9). Results of HE staining and immunofluorescent staining of OC indicate that the tissue-engineered bone had osteogenic differentiation potential under in vivo.

Bone tissue in vivo has a 3D structure and the localization of cells is uniform. Uniform cell density avoids rapid increase in cell density and hence all cells might receive sufficient nutrients. In this study, the blood of rat subcutaneous tissue might provide nutrients for the RBMCs to grow. Tissue-engineered bone combined with AFS and RFB achieved uniform cell distribution in the constructed 3D scaffold. Therefore, the tissue-engineered bone may have bone-forming ability even under in vivo. In addition,

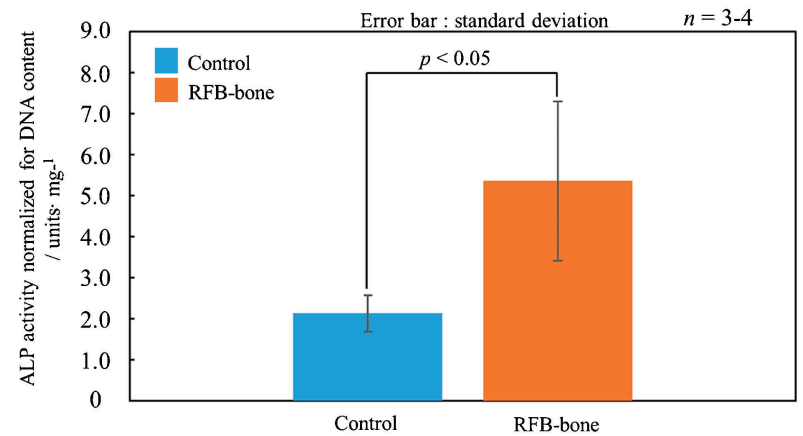

Fig. 8. ALP activity assay normalized for the amount of DNA of the constructed tissue-engineered bones (RFB-bone) and AFS2000 (control)* *: The Mann-Whitney U-test and Z test were carried out with a significance level of $p<0.05$.

implantation of osteodifferentiated cells might have an added advantage in bone formation in vivo. In our previous study, bone formation was confirmed in vivo when AFS and pre-osteodifferentiated cells were implanted. ${ }^{24)}$ Ohgushi et al. ${ }^{25)}$ conducted an experiment to evaluate the bone-forming ability of HAp ceramics cultured with RBMCs by implanting them at rat subcutaneous tissue. As a result, the formation of new bone was confirmed in the ceramics. In addition, similar results have been obtained implantation of osteodifferentiated cells and scaffolds into rat subcutaneous tissue. ${ }^{12)}$ Yoshikawa et al. ${ }^{12)}$ reported effective in vivo bone formation in subcutaneous tissue by implanting osteodifferentiated cells and HAp ceramics. Moreover, the $3 \mathrm{D}$ culture of cells using RFB showed bone differentiation possibly induced by mechanical stimulation in the reactor. ${ }^{21)}$ In summary, it was confirmed that the tissue-engineered bone derived from AFS had a high osteogenic differentiation potential in vivo in the absence of osteoblasts. 

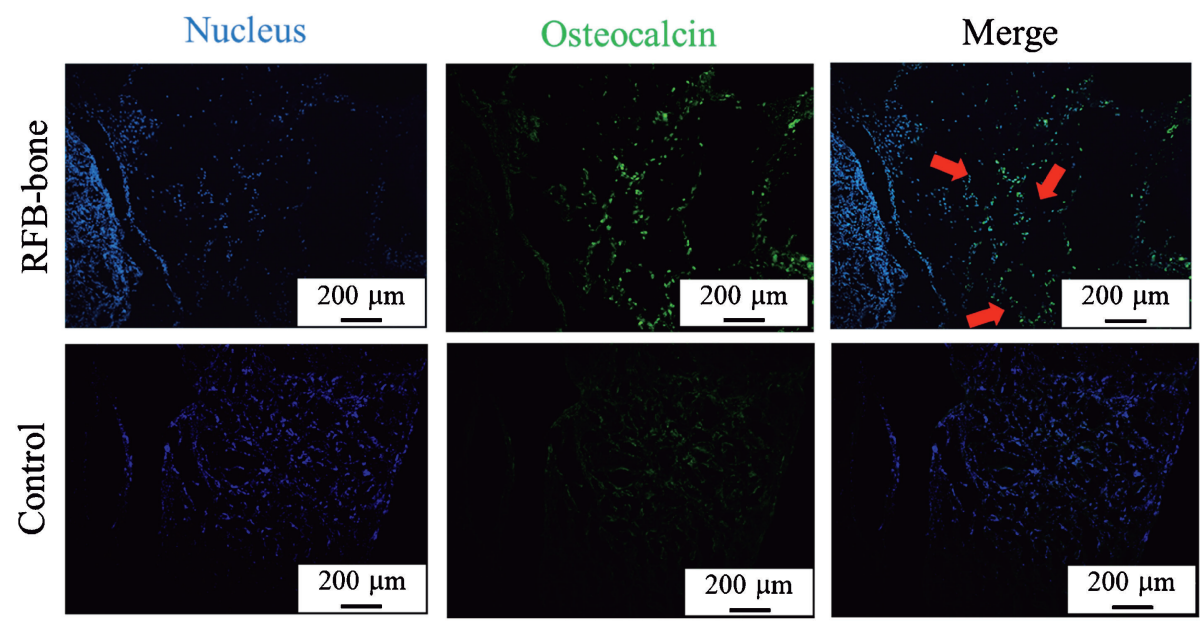

Fig. 9. Immunofluorescence staining of $\mathrm{OC}$ for specimens harvested from subcutaneous tissue of rats. Red arrows: Cells expressing OC.

\section{Conclusion}

A 3D tissue-engineered bone was constructed using AFSs and cultured in an RFB to assess potential clinical applications. Firstly, we established the advantages of RFB for $3 \mathrm{D}$-cell culture. Secondly, to confirm the bone-forming ability, the constructed tissue-engineered bone was implanted subcutaneously in rat tissue where bone formation does not occur naturally due to the absence of osteoblasts and subsequently evaluated using various biological evaluations. We conclude that RFB provides uniform cell distribution and promotes bone differentiation properties. Furthermore, the tissue-engineered bone had a high osteogenic differentiation potential even in the absence of osteoblasts in vivo. This high differentiation potential may lead to a high bone-forming ability. These findings help further our knowledge in bone reconstruction and bone disease treatment using tissue engineering approaches.

\section{References}

1) R. Dimitriou, G. I. Mataliotakis, A. G. Angoules, N. K. Kanakaris and P. V. Giannoudis, Injury, 42 [Suppl 2], S3-S15 (2011).

2) A. R. Vaccaro, Orthopedics, 25, s571-s578 (2002).

3) T. Kurien, R. G. Pearson and B. E. Scammell, Bone Joint J., 95-b, 583-597 (2013).

4) P. Ma, W. Wu, Y. Wei, L. Ren, S. Lin and J. Wu, Mater. Design, 207, 1-11 (2021).

5) H. S. Sohn and J. K. Oh, Biomater. Res., 23, 1-7 (2019).

6) Y. Wang, W. Zhang and Q. Yao, J. Orthop. Transl., 29, 60-71 (2021).

7) J. M. Holzwarth and P. X. Ma, Biomaterials, 32, 96229629 (2011).

8) R. Saito, Y. Ishii, R. Ito, K. Nagatsuma, K. Tanaka, M. Saito, H. Maehashi, H. Nomoto, K. Ohkawa, H. Mano, M. Aizawa, H. Hano, K. Yanaga and T. Matsuura, Artif. Organs, 35, 80-83 (2011).

9) X. Su, T. Wang and S. Guo, Regen. Ther., 16, 63-72
(2021).

10) L. E. Rustom, T. Boudou, S. Lou, I. Pignot-Paintrand, B. W. Nemke, Y. Lu, M. D. Markel, C. Picart and A. J. Wagoner Johnson, Acta Biomater., 44, 144-154 (2016).

11) M. Miura, J. Fukasawa, Y. Yasutomi, H. Maehashi, T. Matsuura and M. Aizawa, Key Eng. Mat., 493-494, 878-883 (2012).

12) T. Yoshikawa, H. Ohgushi, H. Nakajima, E. Yamada, K. Ichijima, S. Tamai and T. Ohta, Transplantation, 69, 128-134 (2000).

13) M. Aizawa, F. S. Howell, K. Itatani, Y. Yokogawa, K. Nishizawa, M. Toriyama and T. Kameyama, J. Ceram. Soc. Jpn., 108, 249-253 (2000).

14) M. Aizawa, A. E. Porter, S. M. Best and W. Bonfield, Biomaterials, 26, 3427-3433 (2005).

15) M. Aizawa, H. Shinoda, H. Uchida, I. Okada, T. J. Fujimi, N. Kanzawa, H. Morisue, M. Matsumoto and Y. Toyama, Phosphorus Res. Bull., 17, 262-268 (2004).

16) M. Miura, J. Fukasawa, Y. Yasutomi, H. Maehashi, T. Matsuura and M. Aizawa, Key Eng. Mat., 529-530, 397-401 (2013).

17) C. Maniatopoulos, J. Sodek and A. H. Melcher, Cell Tissue Res., 254, 317-330 (1988).

18) S. Aoki, J. Anal. Bio-Sci., 28, 119-125 (2005).

19) H. Nomoto, H. Maehashi, M. Shirai, M. Nakamura, T. Masaki, Y. Mezaki, J. Park, M. Aizawa, K. Ohkawa, K. Yoshida and T. Matsuura, Hum. Cell, 32, 1-11 (2019).

20) M. Sila-Asna, A. Bunyaratvej, S. Maeda, H. Kitaguchi and N. Bunyaratavej, Kobe J. Med. Sci., 53, 25-35 (2007).

21) F. W. Janssen, J. Oostra, A. Oorschot and C. A. van Blitterswijk, Biomaterials, 27, 315-323 (2006).

22) R. M. Schwartz, B. O. Palsson and S. G. Emerson, P. Natl. Acad. Sci. USA, 88, 6760-6764 (1991).

23) S. M. Tanaka, J. Biomech. Sci. Eng., 5, 635-645 (2010).

24) K. Suzuki, K. Nagata, T. Yokota, M. Honda and M. Aizawa, Bio-Med. Mater. Eng., 28, 57-64 (2017).

25) H. Ohgushi, Y. Dohi, S. Tamai and S. Tabata, J. Biomed. Mater. Res., 27, 1401-1407 (1993). 